\title{
DEPDC7 inhibits cell proliferation, migration and invasion in hepatoma cells
}

\author{
ZHIJUN LIAO ${ }^{1}$, XINRUI WANG ${ }^{1}$, XIAOJIANG WANG ${ }^{1,2}$, LISHENG LI $^{1}$ and DEXIN LIN ${ }^{1}$ \\ ${ }^{1}$ Department of Biochemistry and Molecular Biology, School of Basic Medical Sciences, Fujian Medical University, Fuzhou, \\ Fujian 350122; ${ }^{2}$ Laboratory of Molecular Pathology, Fujian Provincial Cancer Hospital, Fuzhou, Fujian 350014, P.R. China
}

Received December 27, 2015; Accepted August 11, 2017

DOI: $10.3892 / \mathrm{ol} .2017 .7128$

\begin{abstract}
DEP Domain Containing 7 (DEPDC7) is highly and specifically expressed in normal liver tissue, belonging to the class of genes of liver-selective cell communication. Although the function of DEPDC7 remains poorly understood, its expression is decreased in liver cancer compared with normal liver tissues. It has previously been demonstrated that knockdown of DEPDC7 promotes cell growth, S phase entry and cell mobility and invasion in HepG2 cells. In the present study, it was shown that DEPDC7 expression is downregulated in four hepatoma cell lines (SMMC-7721, Huh-7, SK-Hep-1 and HepG2) and 48 hepatoma tissues, determined using western blot and immunohistochemical analysis. When DEPDC7 is overexpressed in hepatoma cell lines (SK-Hep-1 and Huh-7), it inhibits cell proliferation and cell growth; inhibits cell cycle entry; and inhibits cell motility and invasion. These results, together with the results of knockdown experiments, demonstrate that DEPDC7 may have an important role in hepatoma cells growth and metastasis and suggest it could be a therapeutic target; however, in vitro studies are required to validate this hypothesis.
\end{abstract}

\section{Introduction}

Liver cancer refers primarily to hepatocellular carcinomas (HCC), which accounts for up to 90 percent cases (1) and is the most frequent primary malignancy of liver (2). Despite progress having been achieved in the diagnosis and treatment of hepatoma, it remains among the tumors with the poorest prognosis (3). Previous studies have suggested that alterations in genomics and epigenetics are involved with tumor growth and metastasis, however, the specific mechanisms remain to be

Correspondence to: Professor Dexin Lin, Department of Biochemistry and Molecular Biology, School of Basic Medical Sciences, Fujian Medical University, 1 Xue Yuan Road, University Town, Fuzhou, Fujian 350122, P.R. China

E-mail: dexin9997@163.com

Key words: cell proliferation, DEPDC7, hepatocellular carcinoma, invasion, migration, overexpression elucidated (4). Genomic and epigenetic changes, particularly the accumulation of mutations, affect the normal growth and differentiation of hepatic cells, and thus lead to hepatoma (5). Mutations often break the expression balance of oncogenes and oncogene-suppress genes (6).

DEPDC7 was first identified by Gawin et al in 1999 (7). However, the function of DEPDC7 remains largely unknown. The protein consists of 511 amino acids, with two potentially functional domains consisting of the Dishevelled, EGL-10 and Pleckstrin (DEP) domain and the GTPase activating protein (GAP) domain. The DEP domain is a globular protein domain of 90 amino acids that is present in numerous signaling proteins involved in G-protein signaling pathways and Wnt signaling pathways (8-10). Based on conserved domain analysis and literature mining, it is hypothesized that the proteins containing DEP domain maybe important in cell signal transduction and numerous other biological processes (11). It has previously been shown that DEPDC7 is highly and specifically expressed in normal liver tissue and thus belongs to the class of genes of liver-selective cell communication (LSCC) (12). It was identified that the DEPDC7 gene (NM_001077242.1) is located at chromosome 11p13, where deletion mutations were found in $31.6 \%$ of HCC cells (13). It was therefore considered that chromosome 11p13 may contain genes, such as DEPDC7, that inhibit tumor growth in the liver.

In the present study, the gene expression of DEPDC7 was examined in several hepatoma cell lines, and this was compared with the expression in normal hepatic cells and liver cancer tissues. Wild-type DEPDC7 was overexpressed in two hepatoma cell lines, and cell proliferation, cell cycles progression and cell migration and invasion of these cells were investigated.

\section{Materials and methods}

Cell culture. Human 293 T cells, human hepatic cell L-02, human hepatoma SMMC-7721, Huh-7, SK-Hep-1 and HepG2 cell lines were cultured in Dulbecco's modified Eagle's medium (DMEM; Thermo Fisher Scientific, Inc., Waltham, MA, USA) supplemented with $10 \%$ fetal bovine serum (Gibco; Thermo Fisher Scientific, Inc.), $100 \mathrm{U} / \mathrm{ml}$ penicillin and $100 \mathrm{mg} / \mathrm{ml}$ streptomycin. All cells were maintained at $37^{\circ} \mathrm{C}$ in a humidified atmosphere containing $5 \% \mathrm{CO}_{2}$. Cells were used in studies when they reach $75 \%$ confluence. 
Plasmid construction. The expression vector pLV-EF1 $\alpha$ MCS-IRES-Puro and the helper plasmids pMDLg, pRSV-REV and pVSV-G were kindly provided by Professor Jiahuai Han (School of Life Sciences, Xiamen University, Xiamen, China). The DEPDC7 was amplified using a human cDNA derived from the L-02 liver cells as a template and the following primers: BamHI-F-depdc7, 5'AGAGAATTCGGATCCATG GCCACCGTGCAG3'; SmaI-R-DEPDC7, 5'TGGCTCGAG CCCGGGTCAGTCTCAAAATGCTCA3'. The BamHI and SmaI sites are underlined. The ATG translation initiation site and the TCA termination site of DEPDC7 are shown in bold. Subsequently, the amplified product was cloned into the BamHI and SmaI sites of the vector pLV-EF1 $\alpha$-MCS-IRES-Puro using the Exonuclease III-assisted ligase-free cloning method as previously described (14). All clonal products were identified by BamHI and SmaI and verified by DNA sequencing (Sangon Biotech Co., Ltd., Shanghai, China). The empty vector was used as a negative control for infection and subsequent detection.

Virus packaging and infection. Both recombinant lentiviruses and negative control were packaged in $293 \mathrm{~T}$ cells in the presence of helper plasmids (pMDLg, pRSV-REV and pVSV-G) using TurboFect Infection Reagent (Thermo Fisher Scientific, Inc.), according to the manufacturer's protocol. The infected cells were cultured at $37^{\circ} \mathrm{C}$ for $48 \mathrm{~h}$ and the virus was then collected for infection. Huh-7 and SK-Hep-1 were seeded in a 12 -well cell culture plate, grown overnight at $37^{\circ} \mathrm{C}$ to achieve $30-40 \%$ confluence, and then infected with the virus in fresh medium containing $10 \mu \mathrm{g} / \mathrm{ml}$ polybrene. After $12 \mathrm{~h}$ of incubation, the culture medium was replaced with fresh medium and the infected cells were harvested at $72 \mathrm{~h}$ after infection and then used in subsequent experiments. All virus packaging and infection experiments were conducted in triplicate.

RNA isolation and reverse-transcription quantitative PCR $(R T-q P C R)$. Total RNA was isolated from the collected cells using TRIzol reagent (TakaraBio, Inc., Otsu, Japan) and reverse-transcribed into cDNA using PrimeScript ${ }^{\mathrm{TM}}$ RT reagent Kit (TakaraBio, Inc.), according to the manufacturer's protocol. RNA expression levels were normalized to an internal control, $\beta$-actin. qPCR was conducted as follows: $95^{\circ} \mathrm{C}$ for $10 \mathrm{~min}$; and $95^{\circ} \mathrm{C}$ for $15 \mathrm{sec}$ and $60^{\circ} \mathrm{C}$ for 1 min for 40 cycles. qPCR were performed on the Step One ${ }^{\mathrm{TM}}$ Real-Time PCR system (Applied Biosystems; Thermo Fisher Scientific, Inc.) using SYBER-Green qPCR Supermix (Roche Diagnostics, Basel, Switzerland). The relative expression level of $D E P D C 7$ was normalized to that of $\beta$-actin by $2^{-\Delta \Delta \mathrm{Cq}}$ cycle threshold method (15). Following primers were used: DEPDC7 forward, 5'-ACCTTCCACTTCTTGACT CCTTAC-3' and reverse, 5'-CGAGAGCCACTCATCTTC CTG-3'; $\beta$-Actin forward, 5'-CGTGCGTGACATTAAGGAGAA G-3' and reverse, 5'-GGAAGGAAGGCTGGAAGAGTG-3'.

SDS-PAGE and Western blot analysis detect DEPDC7. Culture plates were rinsed with ice-chilled PBS. The cells were collected using a plastic cell scraper and lysed in Lysis buffer $(20 \mathrm{mM}$ Tris/ $\mathrm{HCl} \mathrm{pH} 7.5 ; 120 \mathrm{mM} \mathrm{NaCl} ; 1 \mathrm{mM}$ EDTA; 1 mM EGTA; 1\% Triton X-100; 2.5 mM Sodium pyrophosphate; $1 \mathrm{mM} \beta$-Glycerophosphate; $1 \mathrm{mM} \mathrm{Na} \mathrm{VO}_{4}$ ) in the presence of protease inhibitor phenylmethylsulfonyl fluoride. Protein were dissolved in $5 \mathrm{X}$ sample buffer $(0.25 \mathrm{M}$
Tris/HCl pH 6.8; $10 \%$ (w/v) SDS; $50 \%$ glycerol; $0.5 \%$ (w/v) bromophenol blue) and were subsequently denatured at $100^{\circ} \mathrm{C}$ for $10 \mathrm{~min}$. Subsequently, $30 \mu \mathrm{g}$ protein were loaded onto $10 \%$ Tris-Acrylamide gels and electrotransferred onto PVDF membranes. The membranes were blocked in $10 \%$ skimmed milk for $2 \mathrm{~h}$ at room temperature and incubated with primary antibodies against $\beta$-actin at a dilution of 1:8,000 (catalog no. 3700; Cell Signaling Technology, Inc., Danvers, MA, USA) and DEPDC7 at a dilution of 1:1,000 (catalog no. ab174659, Abcam, Cambridge, UK) at $4^{\circ} \mathrm{C}$ overnight. Subsequent to washing, the membranes were incubated in horse radish peroxidase (HRP)-conjugated goat anti-mouse/anti-rabbit IgG secondary antibody at a dilution of 1:10,000 (catalog nos. 7076-mouse and 7074-rabbit; Cell Signaling Technology, Inc.) at room temperature for $2 \mathrm{~h}$, with three washes after incubations. Signals were detected using enhanced chemiluminescence (WBKLS0100; EMD Millipore, Billerica, MA, USA) and captured by the ImageQuant LAS 4000 mini Imaging System (GE Healthcare, Chicago, IL, USA).

\section{Immunohistochemical analysis and assessment}

Tissues. A total of 48 hepatoma tissues were used and they were collected between April 2012 and July 2014. The present study was approved by the Ethics Committee of Fuzhou Dongfang Hospital (Fuzhou, China), and the experiments were conducted in accordance with the Declaration of Helsinki and Good Clinical Practice. Informed consent was obtained from all patients prior to the use of tissues.

DEPDC7 immunostaining. DEPDC7 immunohistochemical staining of hepatoma tissues was performed using EliVision ${ }^{\mathrm{TM}}$ plus Polymer HRP IHC kit (Fuzhou Maixin Biotech Co., Ltd., Fuzhou, China). Briefly, Formalin-fixed paraffin-embedded sections were deparaffinized in xylene and dehydrated with ethanol. The tissues were heated in a microwave at $700 \mathrm{~W}$ for $5 \mathrm{~min}$ in $10 \mathrm{mM}$ citrate solution, for antigen retrieval. Endogenous peroxidase activity was blocked by $3 \%$ hydrogen peroxide in methanol for $10 \mathrm{~min}$ at room temperature. The sections were then incubated overnight at $4^{\circ} \mathrm{C}$ with anti-human DEPDC7 (dilution, 1:30; catalog no. HPA015800; Sigma-Aldrich; Merck KGaA, Darmstadt, Germany) in PBS containing $1 \%$ bovine serum albumin. The slides were rinsed and incubated at room temperature with HRP-conjugate anti-mouse IgG (dilution, 1:3,000; catalog no. W4021; Promega Corporation; Madison, WI, USA) for $30 \mathrm{~min}$. The immunoreactivity was visualized with diaminobenzidine (DAB Kit, MAX-001; Fuzhou Maixin Biotech Co., Ltd.). The sections were counter stained with hematoxylin at room temperature for $2 \mathrm{~min}$, dehydrated and evaluated under OLYMPUS BX51 light microscope (Olympus, Tokyo, Japan). For quantification, the intensity of DEPDC7 immunoreactivity was scored ' \pm ', '+', ' ++ ' or ' +++ ', on the basis of the brown-yellow staining area, where ' \pm ' represented no distinct brown granules and ' +++ ' represented the highest strength.

Ki-67 immunostaining. Ki-67 immunostaining was performed using the aforementioned procedure, using mouse monoclonal antibodies against Ki-67 (Ready-to-Use; catalog no. MAB-0672, Fuzhou Maixin Biotech Co., 
A

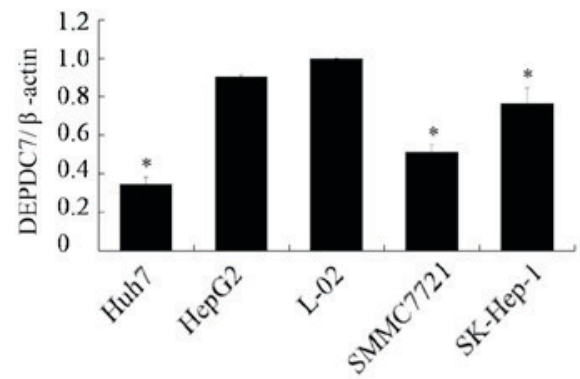

$\mathrm{C}$

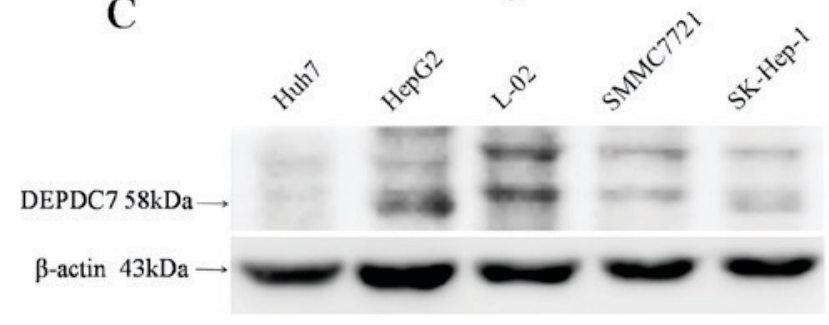

E

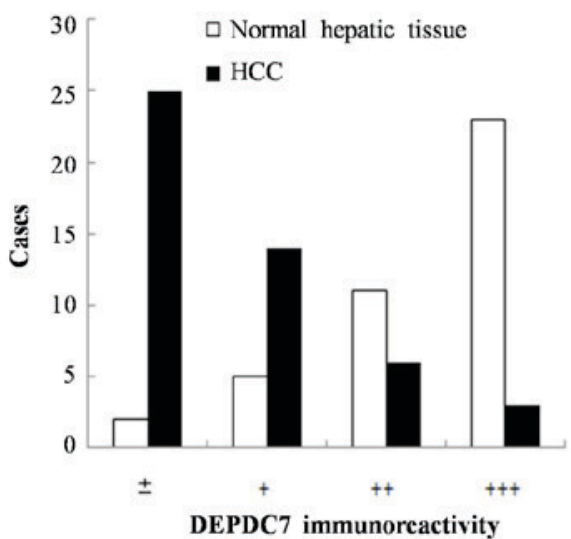

B

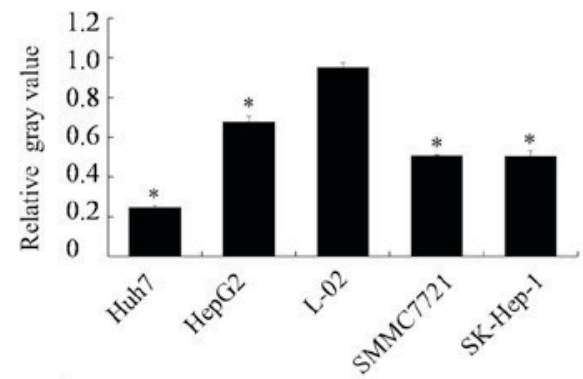

$\mathrm{D}$

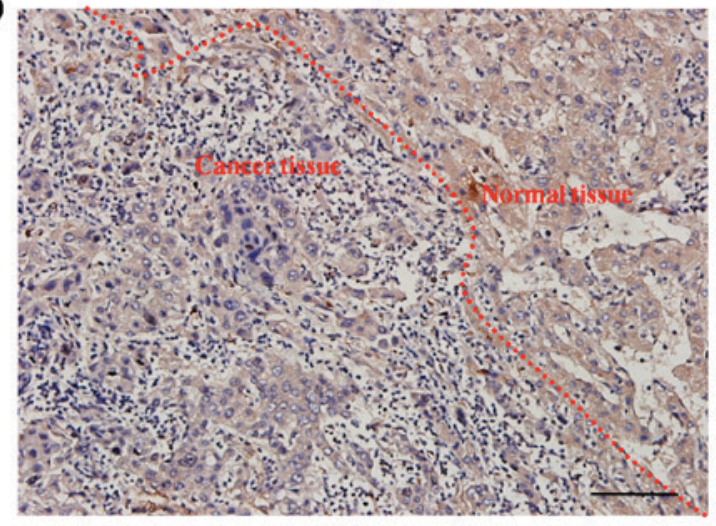

Figure 1. DEPDC7 expression in hepatoma cell lines and tissues. (A) mRNA expression of DEPDC7 in the normal hepatic L-02 cell line and four hepatoma SMMC-7721, HepG2, SK-Hep-1 and Huh-7 cell lines. The expression levels were normalized to $\beta$-actin. Data are expressed as the mean \pm standard from 3 experiments. ${ }^{*} \mathrm{P}<0.05$ was determined by ANOVA with Dunnett's post hoc test for comparison to L-02 cells. (B) The gray value of DEPDC7, which was normalized to that of $\beta$-actin in L-02 cells and the four hepatoma cell lines. Data are expressed as the mean \pm standard deviation from 3 experiments. ${ }^{*} \mathrm{P}<0.05$ was determined by ANOVA with Dunnett's post hoc test for comparison to L-02 cells. (C) Western blot detection of DEPDC7 L-02 and four hepatoma cell lines. $\beta$-actin acts as a loading control. (D) Immunohistochemistry of DEPDC7 of hepatoma tissues. The brown-yellowish staining is stronger on the adjacent normal tissue, in the right upper part of the section (adjacent normal tissue) compared with the left lower part of the section (hepatoma tissue). Scale bar, $50 \mu \mathrm{m}$. (E) Semi-quantitative observation DEPDC7 immunoreactivity of liver sections from 48 hepatoma cases. DEPDC7, DEP domain-containing 7; ANOVA, analysis of variance.

Ltd.). Staining was classified as positive if granular brown-yellow-colored immunoreactivity was present in the nuclei. For quantification, the number of positive cells of total cells was counted.

MTT assay and colony formation assay. Cell proliferation was measured by MTT (Sigma-Aldrich; Merck KGaA) and colony formation assay.

MTT assay. Both of infected and negative control (NC) hepatoma cells were seeded onto 96 -well plates at a density of $2 \times 10^{3}$ cells/well and incubated at $37^{\circ} \mathrm{C}$ in a $5 \% \mathrm{CO}_{2}$ atmosphere for 1-8 days, as previously described (16). For every $24 \mathrm{~h}$, cells were incubated with $20 \mu \mathrm{l}$ of MTT solution at a final concentration of $0.5 \mathrm{mg} / \mathrm{ml} \mathrm{MTT}$ for $4 \mathrm{~h}$. After removing supernatant, $150 \mu \mathrm{l}$ of dimethyl sulfoxide was added to solubilize the formazan salt. After $10 \mathrm{~min}$, the optical density was measure at $570 \mathrm{~nm}$ using a microplate reader (BioTekChina, Beijing, China).
Colony formation assay. In total, 200 cells were plated into each well of 6 -well plate and cultured at $37^{\circ} \mathrm{C}$ for 2 weeks. The cell colonies were stained with crystal violet for $15 \mathrm{~min}$ after fixation in methanol at room temperature. Images of the colonies were captured and the number of colonies in each well was counted, as previously described (17).

Cell cycle assay. Cultured cells were harvested by trypsinization and washed three times in PBS ( $\mathrm{pH} 7.4)$, followed by fixation with precooled $70 \%$ ethanol overnight at $4^{\circ} \mathrm{C}$. Subsequent to centrifugation $\left(1,000 \mathrm{x} \mathrm{g}, 4^{\circ} \mathrm{C}, 5 \mathrm{~min}\right)$ and re-suspension in PBS, the cells were incubated with $50 \mu \mathrm{g} / \mathrm{ml}$ propidium iodide (Sigma-Aldrich; Merck KGaA) and $10 \mu \mathrm{g} / \mathrm{ml}$ RNaseA (Sigma-Aldrich; Merck KGaA) for $30 \mathrm{~min}$ in the dark at $4^{\circ} \mathrm{C}$. The cell cycle was assessed by flow cytometry (FACSVerse; BD Biosciences, Franklin Lakes, NJ, USA) with DRAQ5 $^{\text {TM }}$ Fluorescent Probe (Invitrogen; Thermo Fisher Scientific, Inc.) staining. The data were analyzed using ModFit LT3.0 software (BD Biosciences). 

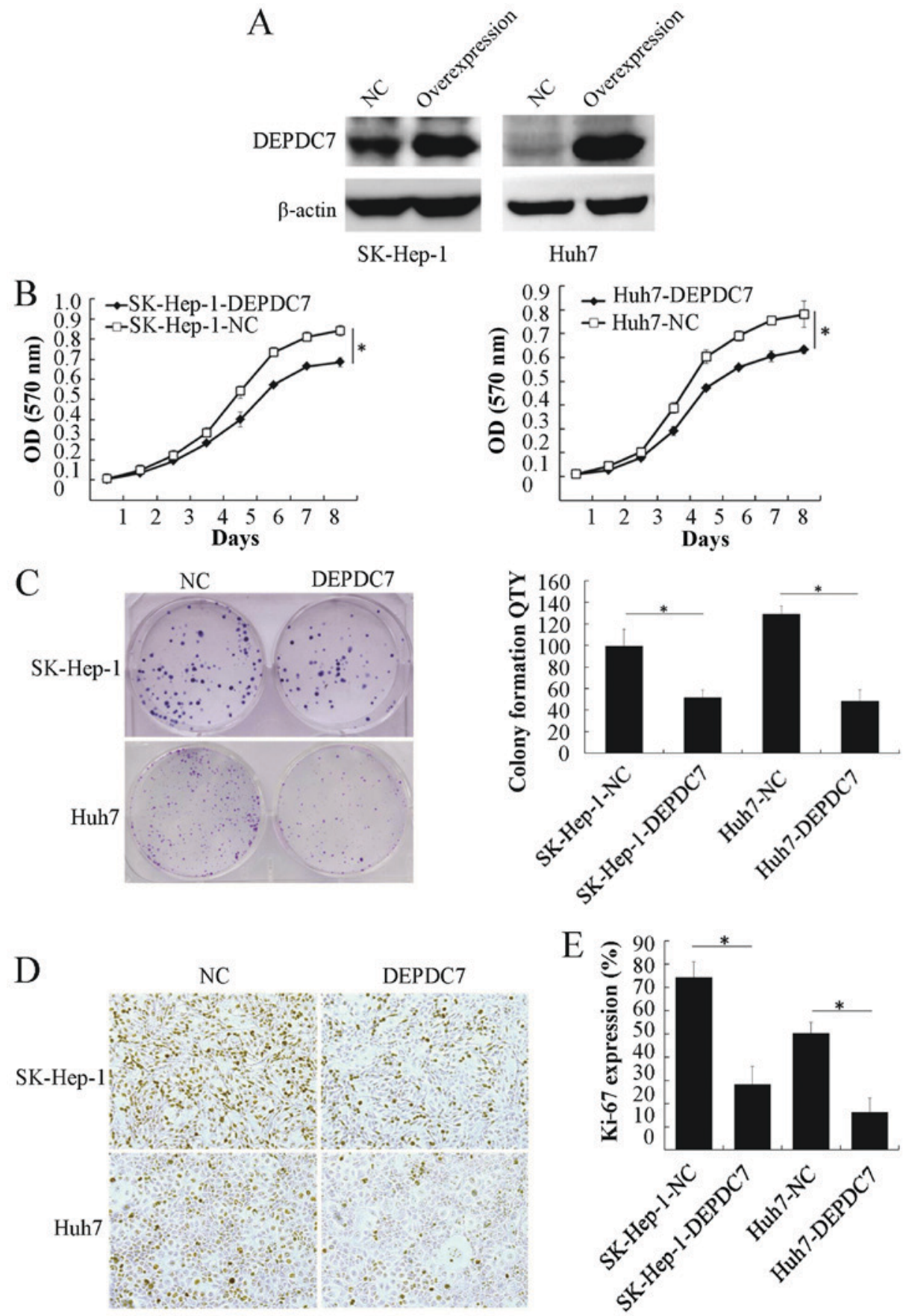

Figure 2. DEPDC7 overexpression inhibits cell proliferation and colony formation. (A) Western blot analysis of DEPDC7-transfected SK-Hep-1 and Huh7 cells and NC cells. (B) Cell growth curve of SK-Hep-1-DEPDC7, Huh7-DEPDC7 and NC cells. Data are expressed as the mean \pm standard deviation from 3 experiments "P<0.05. (C) Colony formation assay of SK-Hep-1-DEPDC7 or Huh7-DEPDC7 cells and NC cells (left panel). The right panel shows colony number of three experiments. Data are expressed as the mean \pm standard deviation. " $\mathrm{P}<0.05$ when compared with the negative control. (D) Immunochemical staining for Ki-67 in the SK-Hep-1 or Huh7 cells with or without infection of plv-DEPDC7. The granular brown-yellow-colored immunoreactivity is evidently increased in the NC groups compared with the DEPDC7 overexpression groups. (E) A semi-quantitative analysis of Ki-67 immunochemistry. Data are expressed as the mean \pm standard deviation from 3 experiments. ${ }^{*}<<0.05$. DEPDC7, DEP domain-containing 7; NC, negative control.

Cell migration and invasion assay. Migration assays were performed using a 24-well Transwell chamber system (Costar 3422; Corning Incorporated, Corning, NY, USA). Cells were seeded in the upper chambers at density of $1 \times 10^{4}$ cells $/ \mathrm{ml}$ in $0.1 \mathrm{ml}$ serum-free DMEM. The lower chambers were filled with $0.8 \mathrm{ml} \mathrm{DMEM}$ with $10 \%$ fetal bovine serum. Following incubation for $24 \mathrm{~h}$ at $37^{\circ} \mathrm{C}$ in a $5 \% \mathrm{CO}_{2}$ atmosphere, the cells that migrated through the membrane were fixed with methanol for $15 \mathrm{~min}$, stained with crystal violet stain for $10 \mathrm{~min}$ at room temperature and counted using a microscope at x200 magnification.

Invasion assays were performed using a 24-well Transwell chamber coated with Matrigel (30 $\mu$ l per filter; BD Biosciences), according to the manufacturer's protocol. Cells were seeded on the top of Matrigel-coated invasion chambers in serum-free DMEM $\left(2 \times 10^{4}\right.$ cells/well $)$ and DMEM containing $10 \%$ fetal bovine serum was added to the lower chambers. Subsequent to incubation for $24 \mathrm{~h}$ at $37^{\circ} \mathrm{C}$ in a $5 \% \mathrm{CO}_{2}$ atmosphere, the cells 
A
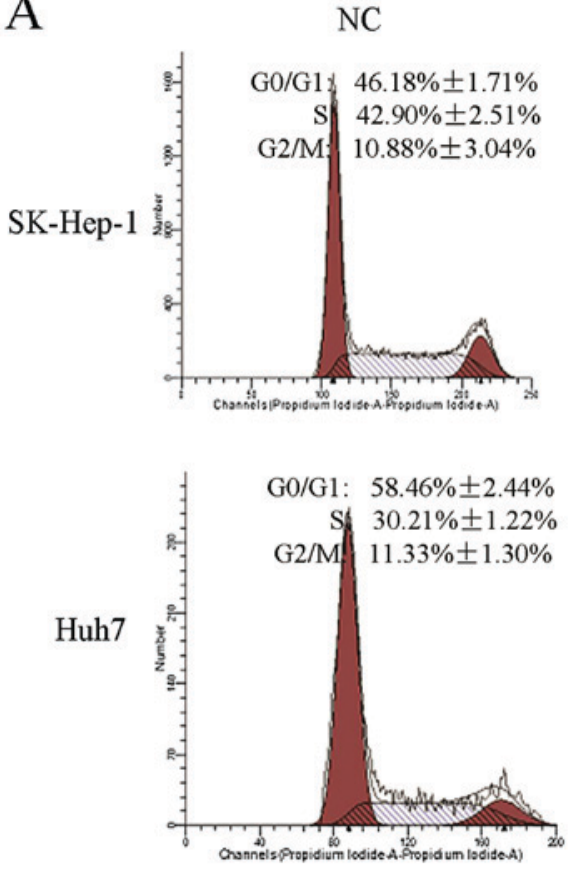
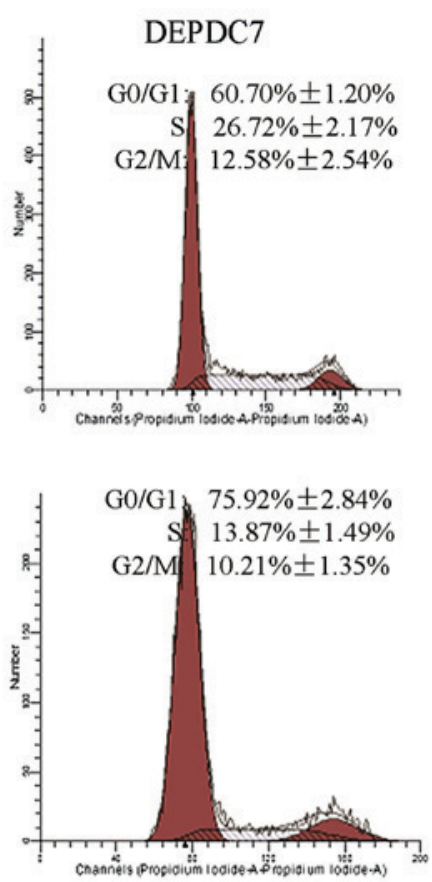
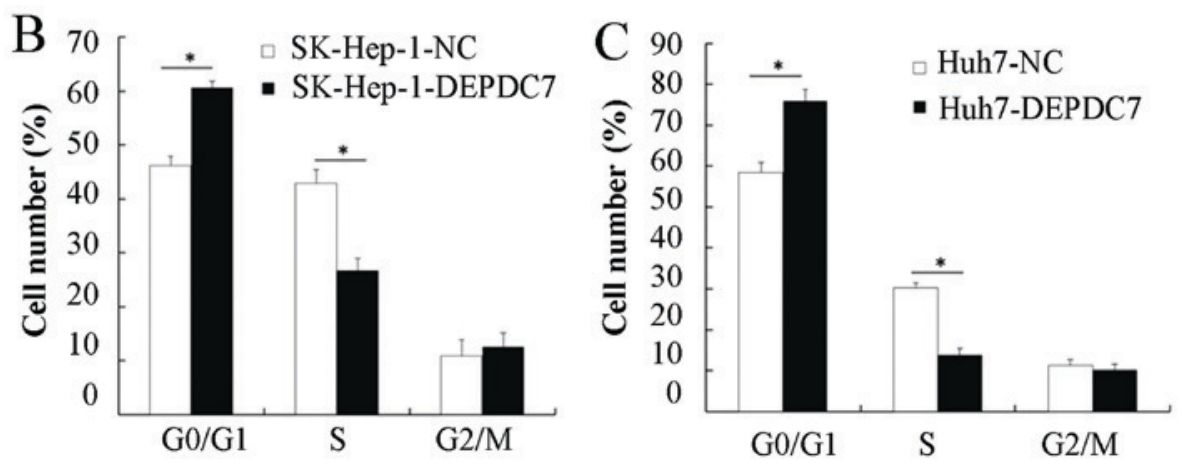

Figure 3. DEPDC7 overexpression leads to cell cycle arrest in hepatoma cells. (A) Cell cycle profiles of SK-Hep-1-DEPDC7, Huh-7-DEPDC7 and NC cells. (B) Distribution of SK-Hep-1 cell numbers in different cycles. Data are expressed as the mean \pm standard deviation from 3 experiments. " $\mathrm{P}<0.05$. (C) Distribution of Huh-7 cell numbers in different cycles. Data are expressed as the mean \pm standard deviation from 3 experiments. ${ }^{*} \mathrm{P}<0.05$. DEPDC7, DEP domain-containing 7; $\mathrm{NC}$, negative control.

that invaded through the membrane were fixed with methanol for $15 \mathrm{~min}$, stained with crystal violet stain for $10 \mathrm{~min}$ at room temperature and counted using a microscope at x200 magnification.

Statistical analysis. The band intensity was quantified using GeneTool 4.01 software (Syngene Inc., Frederick, MD, USA). Statistical analysis was performed using the two-tailed Student's t-test, and independent Student's t-test was used for comparisons of two groups. The significances of the differences between the control and each experimental group was evaluated using one-way analysis of variance and the Dunnett's post hoc test. Data are expressed as the mean \pm standard deviation. $\mathrm{P}<0.05$ was considered to indicate a statistically significant difference.

\section{Results}

DEPDC7 is downregulated in hepatoma cells. Since we have previously found that DEPDC7 is highly and specifically expressed in normal liver tissue (12), it was hypothesized whether the expression of DEPDC7 may be up or downregulated in hepatoma cells. The expression levels of DEPDC7 in all four hepatoma cell lines were significantly decreased compared with those in the normal hepatic L-02 cell line $(\mathrm{P}<0.05$; Fig. 1A-C). The expression of DEPDC7 in hepatoma tissues was also examined. As demonstrated in Fig. 1D, the granular brown-yellow-colored immunoreactivity was evidently weaker in carcinoma cells compared with the surrounding normal hepatic cells. This observation was confirmed in the 41 cases the liver sections obtained hepatoma patients (Fig. 1E). Thus, DEPDC7 is downregulated in the hepatoma cells. Since the Huh-7 and SK-Hep-1 cell lines had the lowest DEPDC7 expression levels, they were used for further functional examination.

Overexpression of DEPDC7 inhibits the proliferation of hepatoma cells. To gain insight into the potential function of DEPDC7, wild-type DEPDC7 was cloned into the pLV-EF1 $\alpha$-MCS-IRES-Puro vector (plv-DEPDC7) and infected into SK-Hep-1 and Huh-7 cell lines (SK-Hep-1-DEPDC7 and Huh-7-DEPDC7). Cells infected 

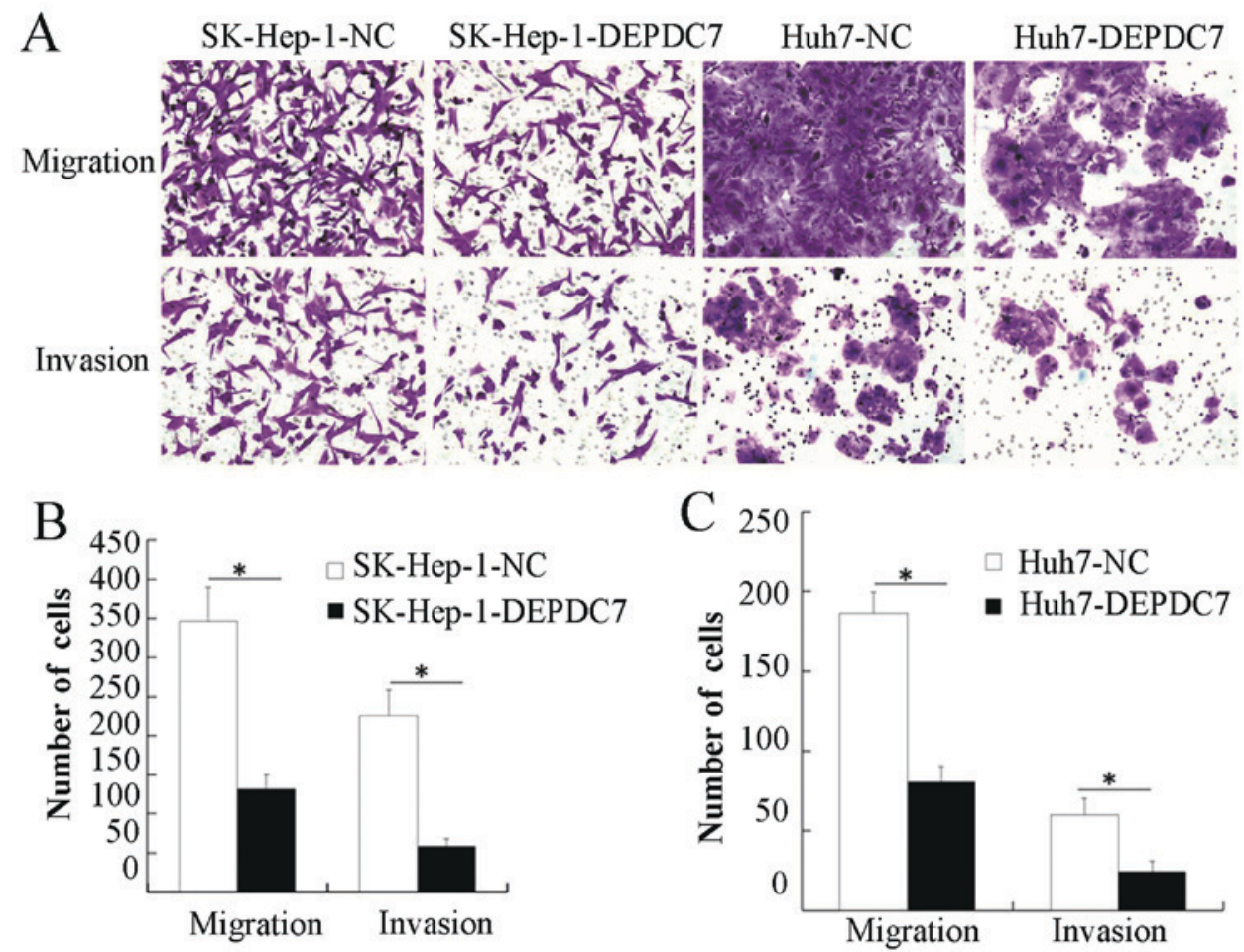

Figure 4. DEPDC7 inhibits migration and invasion of hepatoma cells. (A) SK-Hep-1-DEPDC7, Huh7-DEPDC7 and NC cells that migrated through the membrane or invaded through the Matrigel-coated membrane were stained by crystal violet (magnification, x200). (B and C) Quantification analysis of migration and invasion of (B) SK-Hep-1-DEPDC7 and (C) Huh7-DEPDC7 cells and their respective NC cells. The migrated cells were counted in ten random fields under a light microscope at $x 200$ magnification. Data are expressed as the mean \pm standard deviation from three experiments. ${ }^{*} \mathrm{P}<0.05$. DEPDC7, DEP domain-containing 7 ; NC, negative control.

with empty vector acted as a negative control (SK-Hep-1-NC and Huh-7-NC). The DEPDC7 was found to be significantly overexpressed in these two cell lines compared with NC groups (Fig. 2A). The proliferation of these cells was then examined. After $96 \mathrm{~h}$ of cell seeding, the DEPDC7-transfected cells were significantly decreased in number compared with the $\mathrm{NC}$ groups (Fig. 2B and C), indicating that overexpression of DEPDC7 leads to inhibition of cell proliferation in SK-Hep-1 and Huh-7 cells. The colony formation assay showed that the DEPDC7-overexpressing cells formed fewer colonies than the $\mathrm{NC}$ groups $(\mathrm{P}<0.05$; Fig. $2 \mathrm{D}$ and $\mathrm{E})$, confirming that growth is inhibited in these two hepatoma cell lines when DEPDC7 is overexpressed. Since Ki-67 is a cell proliferation marker (18), $\mathrm{Ki}-67$ expression was examined by immunohistochemistry in SK-Hep-1-DEPDC7, Huh-7-DEPDC7 cells and NC cells. The number of Ki-67-positive cells was significantly decreased in the DEPDC7-overexpression groups compared with the NC groups (Fig. 2D and E). These data demonstrate that DEPDC7 inhibits the development of hepatoma through accelerated cell proliferation.

Overexpression of DEPDC7 blocks the cell cycle transition from the G0/G1 to the $S$ phase. To further investigate the mechanisms of DEPDC7 involved in regulating hepatoma cell proliferation, flow cytometry was used to analyze cell cycles distribution of these cells. As shown in Fig. 3A, the proportion of cells in the G0/G1 phase in both SK-Hep-1-DEPDC7 and Huh7-DEPDC7 cells was increased compared with NC cells, whereas the proportion of cells in the $\mathrm{S}$ phase was decreased in SK-Hep-1-DEPDC7 and Huh7-DEPDC7 cells compared with
$\mathrm{NC}$ cells. The results were consistent in three independent experiments (Fig. 3B and C), indicating that the overexpression of DEPDC7 induced cell cycle arrest in hepatoma cells at the $\mathrm{G} 1 / \mathrm{S}$ phase.

Overexpression of DEPDC7 inhibits the migration and invasion of hepatoma cells. Migration and invasion assays were then performed, to determine whether DEPDC7 has a functional significance on hepatoma cells. SK-Hep-1-DEPDC7, Huh7-DEPDC7 cells and NC cells were grown in Transwell plates, either with or without Matrigel. When the cells that passed through the membrane or Matrigel-coated membrane were stained and counted, SK-Hep-1-DEPDC7 and Huh7-DEPDC7 showed significantly decreased invasion and migration compared with NC cells (Fig. 4), indicating that overexpression of DEPDC7 led to inhibition of the cell motility and cell invasion of hepatoma cells.

\section{Discussion}

The present authors have previously researched liver-specific genes and looked for new targets in the treatment of hepatoma. A previous study indicates that DEPDC7 is highly and specifically expressed in normal liver tissue and thus belongs to the class of genes of LSCC (12). It was shown that expression of DEPDC7 is suppressed in all hepatoma cell lines, both at mRNA and protein levels, suggesting the regulation is transcriptional. Furthermore, DEPDC7 immunoreactivity is frequently decreased in hepatoma tissues compared with normal surrounding tissues. These results suggest that 
DEPDC7 may be involved in the development of hepatoma cells. In a genome-wide molecular profiles research using the Oncomine database, Wurmbach et al (19) also found that DEPDC7 expression is decreased in hepatoma compared with normal liver tissues. It is notable that the expression levels of DEPDC7 vary between hepatoma cell lines and between hepatoma tissues. Further study may investigate the association between DEPDC7 levels and the malignancy of tumors.

Although DEPDC7 has been found for over a decade, the function of it has remained largely unknown. In subsequent functional studies, the role of DEPDC7 gene overexpressed in hepatoma cells was explored, and the results of proliferation and flow cytometry assays illustrated that DEPDC7 overexpression markedly repressed proliferation in hepatoma cell lines by blocking the cell cycle transition between G0/G1 and $\mathrm{S}$ phase, It was also found that the proliferation index reflected by Ki-67 in the DEPDC7-overexpressing hepatocellular carcinoma cells was significantly decreased compared with that in the control hepatocellular carcinoma cells. Additionally, it was further shown that overexpression of DEPDC7 significantly inhibited hepatoma cell migration and invasion. The present results are consistent with our previous findings that the cell proliferation and colony formation are promoted in DEPDC7-knockdown HepG2 cells (20), indicating that DEPDC7 is involved the regulation of cell growth, cell cycle transition and cell mobility and invasion. Although HepG2 was reported to be recognized as a hepatoblastoma rather than a HCC cell line (21), this misidentification did not affect our conclusion for the present study focusing on hepatoma.

In conclusion, the present study suggests that DEPDC7 is a tumor suppress gene. The expression of DEPDC7 is downregulated in hepatoma cells, leading to cell proliferation, cell cycle progression, and migration and invasion. This finding will encourage the identification of the molecular mechanism of DEPDC7-associated inhibition of cell proliferation and motility, and may also encourage the targeting of DEPDC7 as a treatment of hepatoma.

\section{Acknowledgements}

The authors thank Dr Shuo Lin (Biozentrum, University of Basel, Switzerland) for his critical reading and helpful modification of the manuscript. This study was supported by the Foundation for Young Talents of Fujian Province, China (grant no. 2008F3045).

\section{References}

1. Tamai T, Hayato S, Hojo S, Suzuki T, Okusaka T, Ikeda K and Kumada H: Dose finding of lenvatinib in subjects with advanced hepatocellular carcinoma based on population pharmacokinetic and exposure-response analyses. J Clin Pharmacol 57: 1138-1147, 2017.

2. Zhang Q, Zhao S, Pang X and Chi B: MicroRNA-381 suppresses cell growth and invasion by targeting the liver receptor homolog-1 in hepatocellular carcinoma. Oncol Rep 35: 1831-1840, 2016.
3. Simoneau E, Hassanain M, Madkhali A, Salman A, Nudo CG, Chaudhury P and Metrakos P: (18)F-Fluorodeoxyglucose positron-emission tomography could have a prognostic role in patients with advanced hepatocellular carcinoma. Curr Oncol 21: e551-e556, 2014.

4. Patel A and Sun W: Molecular targeted therapy in hepatocellular carcinoma: From biology to clinical practice and future. Curr Treat Options Oncol 15, 380-394, 2014.

5. Chappell G, Kutanzi K, Uehara T, Tryndyak V, Hong HH, Hoenerhoff M, Beland FA, Rusyn I and Pogribny IP: Genetic and epigenetic changes in fibrosis-associated hepatocarcinogenesis in mice. Int J Cancer 134: 2778-2788, 2014.

6. Martincorena I and Campbell PJ: Somatic mutation in cancer and normal cells. Science 349: 1483-1489, 2015.

7. Gawin B, Niederfuhr A, Schumacher N, Hummerich H, Little PF and Gessler M: A 7.5 Mb sequence-ready PAC contig and gene expression map of human chromosome 11p13-p14.1. Genome Res 9: 1074-1086, 1999.

8. $\mathrm{Xu} \mathrm{W}$ and He X: DEEP insights through the DEP domain. Structure 18: 1223-1225, 2010.

9. Consonni SV, Maurice MM and Bos JL: DEP domains: Structurally similar but functionally different. Nat Rev Mol Cell Biol 15: 357-362, 2014.

10. Tauriello DV, Jordens I, Kirchner K, Slootstra JW, Kruitwagen T, Bouwman BA, Noutsou M, Rudiger SG, Schwamborn K, Schambony A, et al: Wnt/beta-catenin signaling requires interaction of the dishevelled DEP domain and $\mathrm{C}$ terminus with a discontinuous motif in frizzled. Proc Natl Acad Sci USA 109: E812-E820, 2012.

11. Wang XR, Yang H and Liao ZJ: Structure and function of the DEP domain. Chemistry of Life 35: 264-271, 2015.

12. Liao ZJ, Ma WL, Liang S, Meng W, Shang T and Zheng WL: Transcriptional regulation of genes involved in liver-selective cell communication. Nan Fang Yi Ke Da Xue Xue Bao 28: 1582-1585, 2008.

13. Kahng YS, Lee YS, Kim BK, Park WS, Lee JY and Kang CS: Loss of heterozygosity of chromosome $8 \mathrm{p}$ and $11 \mathrm{p}$ in the dysplastic nodule and hepatocellular carcinoma. J Gastroenterol Hepatol 18: 430-436, 2003.

14. Li L, Chen W, Liang Y, Ma H, Li W, Zhou Z, Li J, Ding Y, Ren J, Lin J, et al: The Gbetagamma-Src signaling pathway regulates TNF-induced necroptosis via control of necrosome translocation. Cell Res 24: 417-432, 2014.

15. Livak KJ and Schmittgen TD: Analysis of relative gene expression data using real-time quantitative PCR and the 2(-Delta Delta C(T)) method. Methods 25: 402-408, 2001.

16. Meng QC, Wang HC, Song ZL, Shan ZZ, Yuan Z, Zheng Q and Huang XY: Overexpression of NDC80 is correlated with prognosis of pancreatic cancer and regulates cell proliferation. Am J Cancer Res 5: 1730-1740, 2015.

17. Dai J, Wu W, Zhou J, Gao K, Hu G, Lin C, Zhang Y and Li X: Effect of antisense microRNA targeting survivin on rectal cancer HRC-9698 cells and its mechanism. Int J Clin Exp Pathol 8: 6057-6063, 2015.

18. Su H, Ma C, Liu J, Li N, Gao M, Huang A, Wang X, Huang W and Huang X: Downregulation of nuclear receptor FXR is associated with multiple malignant clinicopathological characteristics in human hepatocellular carcinoma. Am J Physiol Gastrointest Liver Physiol 303: G1245-G1253, 2012.

19. Wurmbach E, Chen YB, Khitrov G, Zhang W, Roayaie S, Schwartz M, Fiel I, Thung S, Mazzaferro V, Bruix J, et al: Genome-wide molecular profiles of $\mathrm{HCV}$-induced dysplasia and hepatocellular carcinoma. Hepatology 45: 938-947, 2007.

20. Liao ZJ, Wang XR, Yang $H$ and Zeng YT: Effects of shRNA-mediated knockdown of DEPDC7 on proliferation, migration and invasion of human hepatoma cell HepG2. Chin J Cell Biol 37: 977-983, 2015.

21. Lopez-Terrada D, Cheung SW, Finegold MJ and Knowles BB: Hep G2 is a hepatoblastoma-derived cell line. Hum Pathol 40: $1512-1515,2009$. 